Geometry $\&$ Topology

Volume 6 (2002) 195-218

Published: 27 April 2002

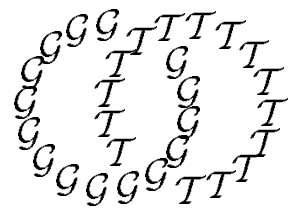

\title{
Homotopy type of symplectomorphism groups of $S^{2} \times S^{2}$
}

\author{
Sílvia ANJos \\ Departamento de Matemática \\ Instituto Superior Técnico, Lisbon, Portugal \\ Email: sanjos@math.ist.utl.pt
}

\begin{abstract}
In this paper we discuss the topology of the symplectomorphism group of a product of two 2-dimensional spheres when the ratio of their areas lies in the interval $(1,2]$. More precisely we compute the homotopy type of this symplectomorphism group and we also show that the group contains two finite dimensional Lie groups generating the homotopy. A key step in this work is to calculate the mod 2 homology of the group of symplectomorphisms. Although this homology has a finite number of generators with respect to the Pontryagin product, it is unexpected large containing in particular a free noncommutative ring with 3 generators.
\end{abstract}

\section{AMS Classification numbers Primary: 57S05, 57R17}

Secondary: $57 \mathrm{~T} 20,57 \mathrm{~T} 25$

Keywords: Symplectomorphism group, Pontryagin ring, homotopy equivalence

Proposed: Yasha Eliashberg

Seconded: Tomasz Mrowka, John Morgan

(C) Geometry $\& \mathcal{G}$ Topology $\mathcal{P}$ ublications
Received: 1 October 2001

Revised: 11 March 2002 


\section{Introduction}

In general symplectomorphism groups are thought to be intermediate objects between Lie groups and full groups of diffeomorphisms. Although very little is known about the topology of groups of diffeomorphisms, there are some cases when the corresponding symplectomorphism groups are more understandable. For example, nothing is known about the group of compactly supported diffeomorphisms of $\mathbb{R}^{4}$, but in 1985, Gromov showed in [4] that the group of compactly supported symplectomorphisms of $\mathbb{R}^{4}$ with its standard symplectic structure is contractible. He also showed that the symplectomorphism group of a product of two 2-dimensional spheres that have the same area has the homotopy type of a Lie group.

More precisely, let $M_{\lambda}$ be the symplectic manifold $\left(S^{2} \times S^{2}, \omega_{\lambda}=(1+\lambda) \sigma_{0} \oplus \sigma_{0}\right)$ where $0 \leq \lambda \in \mathbb{R}$ and $\sigma_{0}$ is the standard area form on $S^{2}$ with total area equal to 1 . Denote by $G_{\lambda}$ the group of symplectomorphisms of $M_{\lambda}$ that act as the identity on $H_{2}\left(S^{2} \times S^{2} ; \mathbb{Z}\right)$. Gromov proved that $G_{0}$ is connected and it is homotopy equivalent to its subgroup of standard isometries $S O(3) \times S O(3)$. He also showed that this would no longer hold when one sphere is larger than the other, and in [9] McDuff constructed explicitly an element of infinite order in $H_{1}\left(G_{\lambda}\right), \lambda>0$. The main tool in their proofs is to look at the action of $G_{\lambda}$ on the contractible space $\mathcal{J}_{\lambda}$ of $\omega_{\lambda}$-compatible almost complex structures.

Abreu and McDuff in [2] calculated the rational cohomology of these symplectomorphism groups and confirmed that these groups could not be homotopic to Lie groups. In particular they computed the cohomology algebra $H^{*}\left(G_{\lambda}, \mathbb{Q}\right)$ for every $\lambda$. For each integer $\ell \geq 1$ we have

$$
H^{*}\left(G_{\lambda} ; \mathbb{Q}\right)=\Lambda(t, x, y) \otimes \mathbb{Q}\left[w_{\ell}\right], \text { when } \ell-1<\lambda \leq \ell
$$

where $\Lambda(t, x, y)$ is an exterior algebra over $\mathbb{Q}$ with generators $t$ of degree 1 , and $x, y$ of degree 3 and $\mathbb{Q}\left[w_{\ell}\right]$ is the polynomial algebra on a generator $w_{\ell}$ of degree $4 \ell$ that is made from $x, y$, and $t$ via higher Whitehead products. The generator $w_{\ell}$ is fragile, in the sense that it disappears (ie, becomes null cohomologous) when $\lambda$ increases. Moreover they showed that the rational homotopy type of $G_{\lambda}$ changes precisely when the ratio of the size of the larger to the smaller sphere passes an integer.

In this paper we show that when $0<\lambda \leq 1$ the whole homotopy type of $G_{\lambda}$ (rather than just its rational part) is generated by its subgroup of isometries $S O(3) \times S O(3)$ and by this new element of infinite order constructed by McDuff. More precisely we will calculate the homotopy type of $G_{\lambda}$ : 
Theorem 1.1 If $0<\lambda \leq 1, G_{\lambda}$ is homotopy equivalent to the product $X=$ $L \times S^{1} \times S O(3) \times S O(3)$ where $L$ is the loop space of the suspension of the smash product $S^{1} \wedge S O(3)$.

In this product of $H$-spaces ${ }^{1}$ one of the $S O(3)$ factors corresponds to rotation in one of the spheres, the other represents the diagonal in $S O(3) \times S O(3)$, and the $S^{1}$ factor corresponds to the generator in $H_{1}\left(G_{\lambda}\right)$ described by Gromov and McDuff. This new element of infinite order represents a $S^{1}$-action that commutes with the diagonal action of $S O(3)$, but not with rotations in each one of the spheres. The loop space $L=\Omega \Sigma\left(S^{1} \wedge S O(3)\right)$ appears as the result of that non-commutativity.

Although this space $X$ is an $H$-space, its multiplication is not the same as on $G_{\lambda}$. This can be seen by comparing the Pontryagin products on integral homology.

The main steps in the proof of this theorem determine the organization of the paper. Therefore in Section 2 we have the first main result which is the calculation of the mod 2 homology ring $H_{*}\left(G_{\lambda} ; \mathbb{Z}_{2}\right)$. Recall that the product structure in $H_{*}\left(G_{\lambda} ; \mathbb{Z}_{2}\right)$, called Pontryagin product, is induced by the product in $G_{\lambda}$. Denote by $\Lambda\left(y_{1}, \ldots, y_{n}\right)$ the exterior algebra over $\mathbb{Z}_{2}$ with generators $y_{i}$ where this means that $y_{i}^{2}=0$ and $y_{i} y_{j}=y_{j} y_{i}$ for all $i, j$, and by $\mathbb{Z}_{2}\left\langle x_{1}, \ldots, x_{n}\right\rangle$ the free noncommutative algebra over $\mathbb{Z}_{2}$ with generators $x_{j}$. Recall that $H_{*}\left(S O(3) ; \mathbb{Z}_{2}\right) \cong \Lambda\left(y_{1}, y_{2}\right)$.

Theorem 1.2 If $0<\lambda \leq 1$ then there is an algebra isomorphism

$$
H_{*}\left(G_{\lambda} ; \mathbb{Z}_{2}\right)=\Lambda\left(y_{1}, y_{2}\right) \otimes \mathbb{Z}_{2}\left\langle t, x_{1}, x_{2}\right\rangle / R
$$

where $\operatorname{deg} y_{i}=\operatorname{deg} x_{i}=i, \operatorname{deg} t=1$ and $R$ is the set of relations $\left\{t^{2}=x_{i}^{2}=\right.$ $\left.0, x_{1} x_{2}=x_{2} x_{1}\right\}$.

The notation implies that $y_{i}$ commutes with $t$ and $x_{i}$. We see that $H_{*}\left(G_{\lambda} ; \mathbb{Z}_{2}\right)$ contains $\Lambda\left(x_{1}, x_{2}\right)$ which appears from rotation in the first sphere, $\Lambda\left(y_{1}, y_{2}\right)$ which represents the diagonal in $S O(3) \times S O(3)$ plus the new generator in $H_{1}\left(G_{\lambda}\right), \lambda>0$, that we denote by $t$. From the inclusion of the subgroup of isometries $S O(3) \times S O(3)$ in $G_{\lambda}$ we have classes $x_{1}, x_{2}, x_{3}=x_{1} x_{2} \in H_{*}\left(G_{\lambda} ; \mathbb{Z}_{2}\right)$ in dimensions 1,2 and 3 respectively, representing the rotation in the first factor. The new generator $t$ in $H_{1}\left(G_{\lambda}\right)$ does not commute with $x_{i}$, therefore we have

\footnotetext{
${ }^{1} X$ is an $H$-space if there is a map $\mu: X \times X \rightarrow X$ such that $\mu \circ i_{1} \simeq 1$ and $\mu \circ i_{2} \simeq 1$ where $i_{1}$ and $i_{2}$ are the inclusions $i_{1}(x)=(x, *)$ and $i_{2}(x)=(*, x), \cong$ means homotopy equivalent and $* \in X$ is a base point.
} 
a nonzero class defined as the commutator and represented by $x_{i} t+t x_{i}$ for $i=1,2,3$. It is easy to understand what these classes are in homotopy. For example, $x_{1}$ is a spherical class, so it represents an element in $\pi_{1}\left(G_{\lambda}\right)$ and $x_{1} t+t x_{1}$ corresponds to the Samelson product $\left[t, x_{1}\right] \in \pi_{2}\left(G_{\lambda}\right)$. This is given by the map

$$
S^{2}=S^{1} \times S^{1} / S^{1} \vee S^{1} \rightarrow G_{\lambda}
$$

induced by the commutator

$$
S^{1} \times S^{1} \rightarrow G_{\lambda}:(s, u) \mapsto t(s) x_{1}(u) t(s)^{-1} x_{1}(u)^{-1} .
$$

Although the mod 2 homology has a finite number of generators with respect to the Pontryagin product we will see it is very large containing in particular a free noncommutative ring on 3 generators, namely the commutators $x_{i} t+t x_{i}$, $i=1,2,3$.

The proof of the theorem generalizes Abreu's work and is based on the fact, proved by Abreu in [1], that the space $\mathcal{J}_{\lambda}$, of almost complex structures on $S^{2} \times S^{2}$ compatible with $\omega_{\lambda}$, is a stratified space with two strata $U_{0}$ and $U_{1}$, where $U_{0}$ is the open subset of $\mathcal{J}_{\lambda}$ consisting of all $J \in \mathcal{J}_{\lambda}$ for which the homology classes $E=\left[S^{2} \times\{p t\}\right]$ and $F=\left[\{p t\} \times S^{2}\right]$ are both represented by $J$-holomorphic spheres and its complement $U_{1}$ is a submanifold of codimension 2. More precisely, $U_{1}$ consists of all $J \in \mathcal{J}_{\lambda}$ for which the homology class of the antidiagonal $E-F$ is represented by a $J$-holomorphic sphere.

In Section 3 we start by giving some considerations about torsion in $H_{*}\left(G_{\lambda} ; \mathbb{Z}\right)$. In particular we establish that $H_{*}\left(G_{\lambda} ; \mathbb{Z}\right)$ has only 2 -torsion. Then we define a map $f$ between $G_{\lambda}$ and the product $X=L \times S \times S O(3) \times S O(3)$ and prove it is in fact an homotopy equivalence. This is obtained from three topological facts: (i) it is enough to find a $\mathbb{Z}$-homology isomorphism from another $H$ space; (ii) a $\mathbb{Z}$-homology isomorphism is implied by an isomorphism with all field coefficients; (iii) homology is computed via Leray-Hirsch for two fibrations of $G_{\lambda}$ over $U_{0}$ and $U_{1}$ and a model space is built using universal properties for maps from loop spaces to topological monoids.

Acknowledgements The results of this paper are part of my doctoral thesis, written at State University of New York at Stony Brook under the supervision of D McDuff. I would like to thank her for all her guidance, advice and support. I also want to thank M Abreu for useful corrections on a version of this paper.

The author acknowledges support from FCT (Fundação para a Ciência e Tecnologia) - Programa Praxis, Fundação Luso-Americana para o Desenvolvimento and Fundação Calouste Gulbenkian. 


\section{The Pontryagin ring $H_{*}\left(G_{\lambda} ; \mathbb{Z}_{2}\right)$}

Recall that for any group $G$ the product $\phi: G \times G \rightarrow G$ induces a product in homology

$$
H_{*}\left(G ; \mathbb{Z}_{2}\right) \otimes H_{*}\left(G ; \mathbb{Z}_{2}\right) \stackrel{\times}{\longrightarrow} H_{*}\left(G \times G ; \mathbb{Z}_{2}\right) \stackrel{\phi_{*}}{\longrightarrow} H_{*}\left(G ; \mathbb{Z}_{2}\right)
$$

called the Pontryagin product, that we will denote by ".". Every time it is clear from the context we will suppress this for simplicity of notation. In this section we will compute the ring structure on $H_{*}\left(G_{\lambda} ; \mathbb{Z}_{2}\right)$ induced by this product. Unless noted otherwise we assume $\mathbb{Z}_{2}$ coefficients throughout.

\subsubsection{Geometric description}

As we mentioned in the introduction, Abreu proved in [1] that if $0<\lambda \leq 1$ the space of almost complex structures compatible with $\omega_{\lambda}, \mathcal{J}_{\lambda}$, is a stratified space with two strata $U_{0}$ and $U_{1}$, where $U_{0}$ is open and dense and $U_{1}$ has codimension 2. $U_{i}$ is the set consisting of all $J \in \mathcal{J}_{\lambda}$ for which the class $E-i F$ is represented by a $J$-holomorphic sphere, where $E$ denotes the homology class of $S^{2} \times\{p t\}$ and $F$ denotes the fiber class $\{p t\} \times S^{2}$. The group of symplectomorphisms $G_{\lambda}$ acts on $\mathcal{J}_{\lambda}$ by conjugation. Moreover the group $G_{\lambda}$ has finite dimensional subgroups $K_{i}$, with $i=0,1$, acting on $M_{\lambda}$, where $K_{0}=S O(3) \times S O(3)$ corresponds to the standard Kähler action of $S O(3) \times S O(3)$ on $S^{2} \times S^{2}$ with complex structure the standard split structure $J_{0}=j_{0} \oplus j_{0}$ and $K_{1}=S O(3) \times S^{1}$ is a Kähler action for a complex structure $J_{1} \in U_{1}$ with the property that the unique $J_{1}$-holomorphic representative $C_{2}$ for the class $E-F$ is fixed by the $S^{1}$ part of the action (see below). The $S O(3)$ part of this action is the same as the diagonal $S O(3)$ action on $S^{2} \times S^{2}$.

The next step is to identify each stratum $U_{i}$ of $\mathcal{J}_{\lambda}$ with the quotient of $G_{\lambda}$ by the isometry group $K_{i}$. The result was proved by Abreu in [1] and is the following:

Proposition 2.1 The stratum $U_{i} \in \mathcal{J}_{\lambda}$ is weakly homotopy equivalent to the quotient $G_{\lambda} / K_{i}, i=0$ or 1 .

Now we can give a brief geometric description of the $S^{1}$ part of the action in $K_{1}$ corresponding to the element of infinite order in $\pi_{1}\left(G_{\lambda}\right)$ constructed by McDuff in [9]. The complex structure $J_{1}$ is tamed by $\omega_{\lambda}$ and the complex manifold $\left(S^{2} \times S^{2}, J_{1}\right)$ is biholomorphic to the projectivization $\mathbf{P}(\mathcal{O}(2) \oplus \mathbb{C})$ 
over $S^{2}$. Here $\mathcal{O}(2)$ is a complex line bundle over $S^{2}$ with first Chern class 2 . This bundle has two natural sections, $\mathbf{P}(\{0\} \oplus \mathbb{C})$ and $\mathbf{P}(\mathcal{O}(2) \oplus\{0\})$, which represent the classes $E+F$ (the diagonal in $S^{2} \times S^{2}$ ) and $E-F$ (the antidiagonal in $\left.S^{2} \times S^{2}\right)$. The element of infinite order in $\pi_{1}\left(G_{\lambda}\right)$ acts on this fibration by rotation on the fibers and leaving fixed the sections corresponding to the classes of the diagonal and antidiagonal. We see that this element is in the stabilizer of $J_{1}$ in $G_{\lambda}$, because this rotation is a complex operation. Moreover for each $J \in U_{0}$ in a neighborhood of $U_{1}$ the action of $t \in \pi_{1}\left(G_{\lambda}\right)$ on $J$ gives a loop around $U_{1}$ which represents the link of $U_{1}$ in $U_{0}$.

\subsection{Relation between $H_{*}\left(G_{\lambda}\right)$ and $H_{*}\left(U_{i}\right)$ : additive version}

The fact that $U_{1}$ is a codimension 2 submanifold of $\mathcal{J}_{\lambda}$ implies that $U_{0}=\mathcal{J}_{\lambda}-U_{1}$ is connected. This means that $G_{\lambda}$ is connected, which in turn implies that $U_{1}$ is also connected. Hence

$$
H_{0}\left(U_{0} ; \mathbb{Z}_{2}\right) \cong \mathbb{Z}_{2} \cong H_{0}\left(U_{1} ; \mathbb{Z}_{2}\right) .
$$

Just as M.Abreu showed in [1] we still have for $p \geq 1$,

$$
H_{p}\left(U_{0} ; \mathbb{Z}_{2}\right) \cong H_{p-1}\left(U_{1} ; \mathbb{Z}_{2}\right) .
$$

This already implies that $H_{1}\left(U_{0} ; \mathbb{Z}_{2}\right) \cong \mathbb{Z}_{2}$. Now consider the following principal fibrations
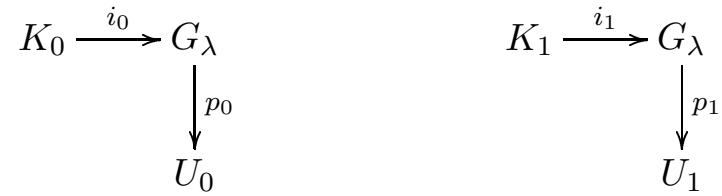

where $K_{i}$ is the identity component of the stabilizer of $J_{i}$ in $G_{\lambda}$. As we stated before $K_{0}$ is the subgroup $S O(3) \times S O(3)$ and $K_{1}$ is isomorphic to $S^{1} \times S O(3)$.

The following proposition was proved by Abreu for rational coefficients but we need it for $\mathbb{Z}_{2}$ coefficients.

Proposition 2.2 Let $\operatorname{Diff}_{0}\left(S^{2} \times S^{2}\right)$ denote the group of diffeomorphisms of $S^{2} \times S^{2}$ that act as the identity on $H_{2}\left(S^{2} \times S^{2}, \mathbb{Z}\right)$. The inclusion

$$
i: K_{0}=S O(3) \times S O(3) \longrightarrow \operatorname{Diff}_{0}\left(S^{2} \times S^{2}\right)
$$

is injective in homology. 
Proof As in [1] we define a map

$$
F: \operatorname{Diff}_{0}\left(S^{2} \times S^{2}\right) \longrightarrow \operatorname{Map}_{1}\left(S^{2}\right) \times \operatorname{Map}_{1}\left(S^{2}\right)
$$

where $\operatorname{Map}_{1}\left(S^{2}\right)$ is the space of all orientation preserving self-homotopy equivalences of $S^{2}$. Given $\varphi \in \operatorname{Diff}_{0}\left(S^{2} \times S^{2}\right)$ we define a self map of $S^{2}$, denoted by $\tilde{\varphi}_{1}$, via the composite

$$
\tilde{\varphi}_{1}: S^{2} \stackrel{i_{1}}{\rightarrow} S^{2} \times S^{2} \stackrel{\varphi}{\rightarrow} S^{2} \times S^{2} \stackrel{\pi_{1}}{\rightarrow} S^{2},
$$

where $i_{1}$, respectively $\pi_{1}$, denote inclusion into, respectively projection onto, the first $S^{2}$ factor of $S^{2} \times S^{2}$. Because $\varphi$ acts as the identity on $H_{2}\left(S^{2} \times\right.$ $\left.S^{2}, \mathbb{Z}\right), \tilde{\varphi}_{1}$ is an orientation preserving self homotopy equivalence of $S^{2}$, ie, $\tilde{\varphi}_{1} \in \operatorname{Map}_{1}\left(S^{2}\right)$. Defining $\tilde{\varphi}_{2}$ in an analogous way using the second $S^{2}$ factor of $S^{2} \times S^{2}$, we have thus constructed the desired map given by

$$
\varphi \mapsto \tilde{\varphi}_{1} \times \tilde{\varphi}_{2} .
$$

It is clear from the construction that $F$ restricted to $S O(3) \times S O(3)$ is just the inclusion

$$
S O(3) \times S O(3) \longrightarrow \operatorname{Map}_{1}\left(S^{2}\right) \times \operatorname{Map}_{1}\left(S^{2}\right)
$$

Now we use the following theorem (see [5]).

Theorem 2.3 The space of orientation preserving self-homotopy equivalences on the 2-sphere has the homotopy type of $S O(3) \times \Omega$, where $\Omega=\tilde{\Omega}_{0}^{2}\left(S^{2}\right)$ is the universal covering space for the component in the double loop space on $S^{2}$ containing the constant based map.

This proves that $S O(3)$ is not homotopy equivalent to $\operatorname{Map}_{1}\left(S^{2}\right)$ but we have, using the Künneth formula with field coefficients,

$$
H_{*}(S O(3) \times \Omega) \cong H_{*}(S O(3)) \otimes H_{*}(\Omega) \cong H_{*}\left(\operatorname{Map}_{1}\left(S^{2}\right)\right)
$$

thus the map

$$
i_{*}: H_{*}(S O(3)) \longrightarrow H_{*}\left(\operatorname{Map}_{1}\left(S^{2}\right)\right)
$$

induced by injection is injective for any field coefficients.

It is proved by D McDuff in [9] that the generator of the $\mathbb{Z}$ factor in $\pi_{1}\left(G_{\lambda}\right)$ lies in $\pi_{1}\left(K_{1}\right)$. This means that the generator of the $S^{1}$-action in $\pi_{1}\left(K_{1}\right)$ maps to a generator of infinite order in $\pi_{1}\left(G_{\lambda}\right)$. Thus the map

$$
i_{1 *}: H_{*}\left(K_{1}\right) \longrightarrow H_{*}\left(G_{\lambda}\right)
$$


induced by inclusion is injective. Since we are working over a field, the cohomology is the dual of homology, thus from the above and Proposition 2.2 the maps

$$
i_{0}^{*}: H^{*}\left(G_{\lambda}\right) \longrightarrow H^{*}\left(K_{0}\right)
$$

and

$$
i_{1}^{*}: H^{*}\left(G_{\lambda}\right) \longrightarrow H^{*}\left(K_{1}\right)
$$

induced by inclusions $i_{0}$ and $i_{1}$ are surjective.

From the Leray-Hirsch Theorem it follows that the spectral sequences of the fibrations collapse at the $E_{2}$-term, and we have the following vector space isomorphisms

$$
\begin{aligned}
& H^{*}\left(G_{\lambda}\right) \cong H^{*}\left(U_{0}\right) \otimes H^{*}\left(K_{0}\right) \\
& H^{*}\left(G_{\lambda}\right) \cong H^{*}\left(U_{1}\right) \otimes H^{*}\left(K_{1}\right) .
\end{aligned}
$$

Passing to the dual we get the homology isomorphisms as vector spaces

$$
\begin{aligned}
& H_{*}\left(G_{\lambda}\right) \cong H_{*}\left(U_{0}\right) \otimes H_{*}\left(K_{0}\right) \\
& H_{*}\left(G_{\lambda}\right) \cong H_{*}\left(U_{1}\right) \otimes H_{*}\left(K_{1}\right) .
\end{aligned}
$$

\subsection{The elements $x_{i}, y_{i}, t$ and $w_{i}$}

Denote by $t$ the generator of infinite order in $H_{1}\left(G_{\lambda} ; \mathbb{Z}\right), \lambda>0$. Recall that $H_{*}(S O(3))=\Lambda\left(x_{1}, x_{2}\right)$ where $\Lambda$ is the exterior algebra on generators $x_{i}$ of degree $i$. Thus $H_{*}\left(K_{0}\right)=\Lambda\left(x_{1}, x_{2}, z_{1}, z_{2}\right)$, where $x_{i}, z_{i}$ represent rotation in first and second factors respectively. The homology of the $S O(3)$ factor in $K_{1} \cong S O(3) \times S^{1}$ is generated by $y_{i}$, and we explain in the next lemma the relation between these generators and the generators $x_{i}$ and $z_{i}$.

Lemma 2.4 The homology ring of the diagonal in $S O(3) \times S O(3), S O_{d}(3)$, is given by $H_{*}\left(S O_{d}(3)\right)=\Lambda\left(y_{1}, y_{2}\right)$ where

$$
\begin{aligned}
& y_{1}=x_{1}+z_{1} \\
& y_{2}=x_{2}+z_{2}+x_{1} z_{1} \\
& y_{3}=x_{3}+z_{3}+x_{1} z_{2}+x_{2} z_{1},
\end{aligned}
$$

$x_{i}$ and $z_{i}$, with $i=1,2$ are the generators of the homology ring of $S O(3) \times$ $S O(3)$ and $x_{3}=x_{1} x_{2}, y_{3}=y_{1} y_{2}$ and $z_{3}=z_{1} z_{2}$. 
Proof It is clear that $y_{i}$ includes terms $x_{i}+z_{i}$, just by looking at the cell structure. Note that the cup product is defined using the diagonal map $d: S O(3) \rightarrow$ $S O(3) \times S O(3)$. If $\alpha \in H^{*}(S O(3))$ generates $H^{1}(S O(3))$ then $(\alpha \cup \alpha)\left(y_{2}\right)=$ $d^{*}(\alpha \times \alpha)\left(y_{2}\right)$. Now we need to define the duals $\hat{x_{1}}$ and $\hat{z_{1}}$ of $x_{1}$ and $z_{1}$ respectively. Let $\hat{x_{1}}$ be the element in $H^{1}(S O(3) \times S O(3))$ such that $\hat{x_{1}}\left(x_{1}\right)=1$, $\hat{x_{1}}\left(x_{i}\right)=0$ if $i=2$ or 3 , and $\hat{x_{1}}\left(z_{i}\right)=0$ if $i=1,2,3$. $\hat{z_{1}}$ is defined in a similar way. We know that the cup product of $\hat{x}_{1}$ and $\hat{z}_{1}$ does not vanish, so we have $0 \neq\left(\hat{x}_{1} \cup \hat{z}_{1}\right)\left(y_{2}\right)$. Hence

$$
\begin{aligned}
\left(\hat{x}_{1} \cup \hat{z}_{1}\right)\left(y_{2}\right) & =d^{*}\left(\hat{x}_{1} \times \hat{z}_{1}\right)\left(y_{2}\right) \\
& =\left(\hat{x}_{1} \times \hat{z}_{1}\right)\left(d_{*} y_{2}\right) \neq 0 .
\end{aligned}
$$

Therefore we see that $d_{*} y_{2}$ must have a component in $H_{1}(S O(3)) \otimes H_{1}(S O(3))$. The only element like that is $x_{1} z_{1}$, so $y_{2}$ must involve this element. The result for $y_{3}$ follows immediately from that for $y_{1}$ and $y_{2}$ by multiplication.

It follows that the generators $y_{i}$ commute with generators $x_{i}$ and $z_{i}$. From injections $i_{0 *}$ and $i_{1 *}$ we have elements $t, x_{i}, z_{i}$ and $y_{i}$ in $H_{*}\left(G_{\lambda}\right)$. From isomorphisms (1) and (5) we know that the rank of $H_{1}\left(G_{\lambda}\right)$ is 3 and as we just showed we have elements $t, x_{1}$ and $y_{1}$ in $H_{1}\left(G_{\lambda}\right)$. Clearly these are linearly independent.

Looking at (5) and (6) we see that $t$ must have a nonzero image in $H_{1}\left(U_{0}\right)$. On the other hand, since the homology of the $S O(3)$ factor in $K_{1}$ is generated by the $y_{i}$, the $x_{i}$ must have a nonzero image in $H_{i}\left(U_{1}\right)$. The class $x_{1}$ must correspond by (1) to a class in $H_{2}\left(U_{0}\right)$ and we will see in Lemma 2.8 below that this class is the image $x_{1} t$ in $U_{0} . x_{1}$ is a spherical representative of the first $S O(3)$ factor in $H_{1}\left(K_{0}\right)$. Therefore, since $K_{0}$ acts on $\mathcal{J}_{\lambda}$ by multiplication on the left there is a well defined $2-$ cycle $x_{1} t$ in $U_{0}$. More precisely if $x_{1}$ is represented by

$$
S^{1} \rightarrow G_{\lambda}: u \mapsto x_{1}(u)
$$

and $t$ by

$$
S^{1} \rightarrow G_{\lambda}: v \mapsto t(v)
$$

we define a $2-$ cycle in $G_{\lambda}$ given by the map

$$
S^{2}=S^{1} \times S^{1} / S^{1} \vee S^{1} \rightarrow G_{\lambda}
$$

induced by the commutator

$$
S^{1} \times S^{1} \rightarrow G_{\lambda}:(v, u) \mapsto t(v) x_{1}(u) t(v)^{-1} x_{1}(u)^{-1} .
$$

It turns out that the projection of this element in $H_{*}\left(G_{\lambda}\right)$ to $H_{*}\left(U_{0}\right)$, under the projection map $p_{0 *}$, is the $2-$ cycle $x_{1} t$ in $U_{0}$. In order to see that let us 
recall that for any group $G$ the Samelson product $[x, y] \in \pi_{p+q}(G)$ of elements $x \in \pi_{p}(G)$ and $y \in \pi_{q}(G)$ is represented by the commutator

$$
S^{p+q}=S^{p} \times S^{q} / S^{p} \vee S^{q} \rightarrow G:(u, v) \mapsto x(u) y(v) x(u)^{-1} y(v)^{-1} .
$$

The Samelson product in $\pi_{*}(G)$ is related to the Pontryagin product in $H_{*}(G ; \mathbb{Z})$ by the formula

$$
[x, y]=x y-(-1)^{|x||y|} y x,
$$

where we suppressed the Hurewicz homomorphism $\rho: \pi_{*}(G) \rightarrow H_{*}(G)$ to simplify the expression. Therefore we see that this $2-$ cycle is given by the commutator $\left[x_{1}, t\right]$, so in homology is simply given by $x_{1} t+t x_{1} \in H_{2}\left(G_{\lambda} ; \mathbb{Z}_{2}\right)$. Similarly we define a cycle in $H_{4}\left(G_{\lambda} ; \mathbb{Z}_{2}\right)$ that in homotopy is given by the commutator $\left[t, x_{3}\right]$. Although $x_{2}$ is not a spherical class, ie, $x_{2} \notin \pi_{2}\left(G_{\lambda}\right)$ we can consider a cycle in degree 3 given by $x_{2} t+t x_{2}$ in $H_{*}\left(G_{\lambda}, \mathbb{Z}_{2}\right)$.

Definition 2.5 We define elements $w_{i} \in H_{i+1}\left(G_{\lambda}, \mathbb{Z}_{2}\right)$ to be the commutators $x_{i} t+t x_{i}$ with $i=1,2,3$. For a word in the $w_{i}^{\prime} s$ we use the notation $w_{I}=$ $w_{i_{1}} \ldots w_{i_{n}}$ with $I=\left(i_{1}, \ldots, i_{n}\right)$.

The reason why we use these classes $x_{i} t+t x_{i}$ instead of simply $x_{i} t, t x_{i}$ is first because they project simultaneously to additive generators in $H_{*}\left(U_{1}\right)$ and $H_{*}\left(U_{0}\right)$ so it is easier to see the correspondence between elements in isomorphisms (5) and (6). Secondly they are in the kernel of the subalgebra of $H^{*}\left(G_{\lambda}\right)$ generated by the duals $\hat{t}$ and $\hat{x}_{i}$ of $t$ and $x_{i}$. We show this fact in the next lemma, but first we define the duals of these elements in $H^{1}\left(G_{\lambda}\right)$. $\hat{t}$ is the element in $H^{1}\left(G_{\lambda}\right)$ such that $\hat{t}(t)=1$ and $\hat{t}\left(x_{1}\right)=\hat{t}\left(y_{1}\right)=0$. We define $\hat{x_{1}}$ and $\hat{y_{1}}$ in the obvious way. We also have $\hat{x_{i}}=\left(\hat{x_{1}}\right)^{i}$ and $\hat{y_{i}}=\left(\hat{y_{1}}\right)^{i}$.

Lemma 2.6 The cup product $\left(\hat{t} \cup \hat{x}_{i}\right)$ evaluated at the commutator $\left[x_{i}, t\right]$ is 0 where $\hat{t}$ and $\hat{x}_{i}$ represent the dual of $t$ and $x_{i}$ in $H^{*}\left(G_{\lambda}\right)$ respectively.

Proof Although in this section we are working with $\mathbb{Z}_{2}$ coefficients we will prove a stronger result by showing that the statement is true also over $\mathbb{Z}$. Note that $\left(\hat{t} \cup \hat{x}_{i}\right)\left(\left[x_{i}, t\right]\right)=\left(\hat{t} \cup \hat{x}_{i}\right)\left(x_{i} t+t x_{i}\right)=\left(\hat{t} \cup \hat{x}_{i}\right)\left(x_{i} t\right)+\left(\hat{t} \cup \hat{x}_{i}\right)\left(t x_{i}\right)$ and we show that $\left(\hat{t} \cup \hat{x}_{i}\right)\left(t x_{i}\right)=\left(\hat{x_{i}} \cup \hat{t}\right)\left(x_{i} t\right)=-\left(\hat{t} \cup \hat{x}_{i}\right)\left(x_{i} t\right)=1$. For example, in the case when $i=1$ consider $f: S^{1} \times S^{1} \rightarrow G_{\lambda}:(t, s) \mapsto \varphi_{t} \psi_{s}$, where $S^{1} \rightarrow G_{\lambda}: t \mapsto \varphi_{t}$ and $S^{1} \rightarrow G_{\lambda}: s \mapsto \psi_{s}$ represent the cycles $t$ and $x_{1}$ respectively. Then

$$
\begin{aligned}
\left(\hat{t} \cup \hat{x_{1}}\right)\left(t x_{1}\right) & =f^{*}\left(\hat{t} \cup \hat{x_{1}}\right)\left[S^{1} \times S^{1}\right] \\
& =f^{*}(\hat{t}) \cup f^{*}\left(\hat{x_{1}}\right)\left[S^{1} \times S^{1}\right] \\
& =f^{*}(\hat{t})\left[S^{1}\right] f^{*}\left(\hat{x_{1}}\right)\left[S^{1}\right]=1 .
\end{aligned}
$$

Thus $\left(\hat{t} \cup \hat{x_{1}}\right)\left(\left[x_{1}, t\right]\right)=-1+1=0$. 
We look at an additive basis for each group $H^{k}\left(G_{\lambda}\right)$ and $H_{k}\left(G_{\lambda}\right)$ in the sense that we want to have a canonical identification between $H^{k}\left(G_{\lambda}\right)$ and $H_{k}\left(G_{\lambda}\right)$, this meaning that if $\left\{c_{\alpha}\right\}$ is an additive basis for $H_{k}\left(G_{\lambda}\right)$ then $\left\{\hat{c}_{\alpha}\right\}$ is an additive basis for $H^{k}\left(G_{\lambda}\right)$ where $\hat{c}_{\alpha}$ is the element satisfying $\hat{c}_{\alpha}\left(c_{\beta}\right)=\delta_{\alpha \beta}$. Using this identification we see from the previous lemma that the dual of the commutators $\left[x_{i}, t\right]$ represent classes in $H^{*}\left(G_{\lambda}\right)$ which are not in the subalgebra of $H^{*}\left(G_{\lambda}\right)$ generated by $\hat{t}, \hat{x}_{i}$ and $\hat{y}_{i}$.

We choose a normalized set of elements in the subring of $H_{*}\left(G_{\lambda}\right)$ generated by $t, x_{i}, y_{j}$ with $i, j=1,2,3$.

Lemma 2.7 Any word in the $t, x_{i}, y_{j}$ with $i, j=1,2,3$ is a sum of elements of the form

$$
w_{I} t^{\epsilon_{t}} x_{i}^{\epsilon_{i}} y_{j}^{\eta_{j}}
$$

where $\epsilon_{t}, \epsilon_{i}, \eta_{j}=0$ or $1, I=\left(i_{1}, \ldots, i_{k}\right)$ and $i, j=1,2$ or $3\left(x_{3}=x_{1} x_{2}, y_{3}=\right.$ $\left.y_{1} y_{2}\right)$.

Proof We know that $y_{j}$ commutes with all other elements and we have equations

$$
\begin{gathered}
x_{i} w_{j}=w_{i} x_{j} \text { if }(i, j) \neq(1,2) \text { and }(2,1) \\
x_{i} w_{j}=w_{i} x_{j}+w_{3} \text { if }(i, j)=(1,2) \text { or }(2,1) .
\end{gathered}
$$

We also know that $x_{i} t=t x_{i}+w_{i}, t$ commutes with $w_{i}$ for $i=1,2,3$ and $t^{2}=0$. These facts together with the two equations imply that we can always bring any copy of $x_{i}$ to the right of $t$ and the $w_{i}{ }^{\prime} s$, adding, if necessary, words on the $w_{i}{ }^{\prime} s$.

\subsection{A generating set for $H_{*}\left(G_{\lambda}\right)$}

In this subsection the aim is to show that the elements $x_{i}, y_{j}, t$ generate the ring $H_{*}\left(G_{\lambda} ; \mathbb{Z}_{2}\right)$. In order to do that we give a geometric description of the isomorphism $H_{p+1}\left(U_{0}\right) \cong H_{p}\left(U_{1}\right)$.

We have projections $p_{i_{*}}: H_{*}\left(G_{\lambda}\right) \rightarrow H_{*}\left(U_{i}\right)$ with $i=0$ or 1 . Since $x_{i}$ has image in $H_{i}\left(K_{0}\right)$ and $t$ has image in $H_{1}\left(K_{1}\right)$ we can conclude that $p_{0 *}\left(\left[x_{i}, t\right]\right)=$ $p_{0 *}\left(x_{i} t\right)$ in $H_{*}\left(U_{0}\right)$ and $p_{1 *}\left(\left[x_{i}, t\right]\right)=p_{1 *}\left(t x_{i}\right)$ in $H_{*}\left(U_{1}\right)$. We write $t$ for $p_{0 *}(t) \in H_{*}\left(U_{0}\right)$ and $x_{i}$ for $p_{1_{*}}\left(x_{i}\right) \in H_{*}\left(U_{1}\right)$. However it will be convenient to distinguish notationally between the different incarnations of $w_{1}, w_{2}, w_{3}$ on the different spaces. We will denote by $v_{i}=p_{0 *}\left(w_{i}\right)$ the generators in $H_{*}\left(U_{0}\right)$ and 
by $u_{i}=p_{1 *}\left(w_{i}\right)$ the generators in $H_{*}\left(U_{1}\right)$ where $i=1,2$ or 3 . Let $v_{I}=p_{0 *}\left(w_{I}\right)$ and $u_{I}=p_{1 *}\left(w_{I}\right)$ where $w_{I}$ is given as in Definition 2.5. This way we give meaning to expressions as $v_{i} v_{j}=p_{0 *}\left(w_{i} w_{j}\right), v_{i} t=p_{0 *}\left(w_{i} t\right)$ and $u_{i} u_{j}=p_{1 *}\left(w_{i} w_{j}\right)$. We can write $v_{i} t$ or $t v_{i}$ to refer to the same element because $t$ commutes with $w_{i}$ in $H_{*}\left(G_{\lambda}\right)$. Note that $H_{*}\left(U_{i}\right)$ is a left $H_{*}\left(G_{\lambda}\right)$-module, so $H_{*}\left(G_{\lambda}\right)$ acts on $H_{*}\left(U_{i}\right)$ by multiplication on the left. Using this module action we have $v_{I^{\prime}}=w_{i} \cdot v_{I}$ and $u_{I^{\prime}}=w_{i} \cdot u_{I}$ for $I^{\prime}=(i, I)$.

We can choose right inverses $s_{i}: H_{*}\left(U_{i}\right) \rightarrow H_{*}\left(G_{\lambda}\right)$ such that $s_{0}(t)=t$, $s_{0}\left(v_{i}\right)=w_{i}, s_{1}\left(x_{i}\right)=x_{i}, s_{1}\left(u_{i}\right)=w_{i}$ and $p_{i_{*}} \circ s_{i}=i d$. They exist because of isomorphisms (5) and (6). Moreover we can choose $s_{i}$ such that $s_{0}$ preserves multiplication by $t, w_{i}$ and $s_{1}$ preserves multiplication by $w_{i}$.

Lemma 2.8 The isomorphism $H_{p+1}\left(U_{0}\right) \cong H_{p}\left(U_{1}\right)$ is given by the map

$$
\psi: H_{p}\left(U_{1}\right) \rightarrow H_{p+1}\left(U_{0}\right): c \mapsto p_{0 *}\left(s_{1}(c) t\right)
$$

Proof Note that since $U_{1}$ is a codimension 2 submanifold of $\mathcal{J}_{\lambda}$, there is a circle bundle $\partial \mathcal{N}_{U_{1}}$ where $\partial \mathcal{N}_{U_{1}}$ is a neighborhood of $U_{1}$ in $U_{0}$ :

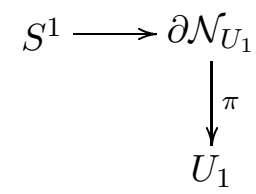

Therefore for any map representing a cycle $c \in H_{p}\left(U_{1}\right)$ we can obtain a cycle in $H_{p+1}\left(U_{0}\right)$ by lifting the map to $\partial \mathcal{N}_{U_{1}}$ using the fibration (8). More precisely, using the section $s_{1}$ we can lift $c$ to a cycle in $H_{*}\left(G_{\lambda}\right)$. This is represented by a map $\tau: C \rightarrow G_{\lambda}: z \mapsto \tau(z)$. Now note that there is a map from the image of $\tau$ to $U_{0}$ given by $g \mapsto g_{*} J$, where $g \in \tau(z)$ and we can choose $J \in U_{0}$ close to $U_{1}$. In fact, we can choose $J \in \mathcal{N}_{U_{1}}$ so close to $U_{1}$ such that $g_{*} J \in \mathcal{N}_{U_{1}}$ also. Then using the $S^{1}$ action, represented by $t$, we define a map to $U_{0}$, representing a cycle in $\mathcal{N}_{U_{1}} \subset U_{0}$ : for each $g$ in the image of $\tau$ we get a loop around $U_{1}$ defined by $g_{*}\left(t_{*} J\right)=(g t)_{*} J$. Therefore the cycle $c$ lifts to $p_{0 *}\left(s_{1}(c) t\right)$ which represents a cycle in $H_{*}\left(U_{0}\right)$.

Remark 2.9 Using the notation introduced above we can write

$$
\begin{aligned}
\psi\left(x_{i}\right) & =p_{0 *}\left(s_{1}\left(x_{i}\right) t\right)=x_{i} t=v_{i}, \\
\psi\left(u_{i}\right) & =p_{0 *}\left(s_{1}\left(u_{i}\right) t\right)=w_{i} t=v_{i} t, \\
\psi\left(u_{I} x_{i}\right) & =w_{I} \cdot v_{i}=v_{I^{\prime}}
\end{aligned}
$$

with $I^{\prime}=(I, i)$ and $\psi\left(u_{I}\right)=v_{I} t$. 
The map $\phi$ that gives the corresponding isomorphism in cohomology,

$$
\phi: H^{p+1}\left(U_{0}\right) \rightarrow H^{p}\left(U_{1}\right),
$$

is the composite of the restriction $i^{*}: H^{*}\left(U_{0}\right) \longrightarrow H^{*}\left(\partial \mathcal{N}_{U_{1}}\right)$ with integration over the fiber of the projection $\pi: \partial \mathcal{N}_{U_{1}} \rightarrow U_{1}$ of the fibration (8) (see [2]).

Now we use the lemma to prove the following proposition.

Proposition 2.10 The generators of the Pontryagin ring $H_{*}\left(G_{\lambda}\right)$ are $t, x_{i}, y_{j}$ with $i, j=1,2$.

Proof The existence of injections $i_{i *}: H_{*}\left(K_{i}\right) \rightarrow H_{*}\left(G_{\lambda}\right)$ with $i=0$ or 1 imply that we have elements $t, x_{i}, y_{j}$ in $H_{*}\left(G_{\lambda}\right)$. Let $R_{*} \subset H_{*}\left(G_{\lambda}\right)$ be the subring generated by $t, x_{i}, y_{j}$. Suppose there is an element of minimal degree $d$ in $H_{*}\left(G_{\lambda}\right)-R_{*}$. From isomorphism (5) we can conclude that such an element would be mapped to a sum of elements

$$
\sum_{l} c_{l} \otimes k_{l} \in \underset{l}{\oplus}\left(H_{d-l}\left(U_{0}\right) \otimes H_{l}\left(K_{0}\right)\right)
$$

with $0 \leq l \leq 6$. For some $l, c_{l}$ is not a polynomial in the $v_{I}, t$. Take the largest such $l$. By the isomorphism in Lemma 2.8 and Remark 2.9 this would create an element in $H_{d-l-1}\left(U_{1}\right)$ that is not a polynomial in $u_{I}$ and $x_{i}$. But this is impossible because this would give rise to a new generator in $H_{d-l-1}\left(G_{\lambda}\right)$ corresponding to this new element in $H_{d-l-1}\left(U_{1}\right) \otimes H_{0}\left(K_{1}\right)$ and this contradicts the minimality of $d$.

\subsection{Main theorem}

We start by showing that we have isomorphisms $H_{*}\left(G_{\lambda}\right) \cong H_{*}\left(U_{i}\right) \otimes H_{*}\left(K_{i}\right)$ given by the Pontryagin product. More precisely, we can define maps

$$
\varphi_{i}: H_{*}\left(U_{i}\right) \otimes H_{*}\left(K_{i}\right) \rightarrow H_{*}\left(G_{\lambda}\right): c \otimes k \mapsto s_{i}(c) . k
$$

with $i=0$ or 1 . Since $K_{i} \subset G_{\lambda}$ and $i_{i}$ is injective in homology we denote $i_{i *}(k)$ simply by $k$. Recall that we have projections $p_{i}: G_{\lambda} \rightarrow U_{i}$ as defined in diagram (2) and these induce maps $p_{i_{*}}: H_{*}\left(G_{\lambda}\right) \rightarrow H_{*}\left(U_{i}\right)$ in homology. It is clear that $p_{i_{*}}\left(s_{i}(c) . k\right)=0$ if $k \in H_{*}\left(K_{i}\right)$, with $*>0$ and the product $s_{0}(c) k$ is an element in the normalized set defined in Lemma 2.7, because $s_{0}(c)$ is a product of $w_{i}^{\prime} s$ and $t$ and $k$ is a product of $x_{i}$ and $y_{j}$. We now claim that these maps are isomorphisms. 
Proposition 2.11 The maps $\varphi_{i}: H_{*}\left(U_{i}\right) \otimes H_{*}\left(K_{i}\right) \rightarrow H_{*}\left(G_{\lambda}\right): c \otimes k \mapsto s_{i}(c) . k$ given by Pontryagin product are isomorphisms.

Proof Consider the elements of the form $v_{I} t^{\epsilon_{t}}$, with $\epsilon_{t}=0,1$ in $H_{*}\left(U_{0}\right)$. If they are not linearly independent, choose a maximal linearly independent subset $B=\left\{c_{\alpha}\right\}$. It follows from Proposition 2.10 that this is a basis for $H_{*}\left(U_{0}\right)$. Now consider the image in $H_{*}\left(G_{\lambda}\right)$ of $B$. This is given by $B^{\prime}=\left\{s_{0}\left(c_{\alpha}\right)\right\}$ with $c_{\alpha} \in B$. These are elements of the form $w_{I} t^{\epsilon_{t}}, \epsilon_{t}=0,1$ and the set $B^{\prime}$ is linearly independent. Therefore it is an additive basis for the space spanned by elements of the form $w_{I} t^{\epsilon_{t}}$. Note that $H_{*}\left(G_{\lambda}\right)$ has a subalgebra isomorphic to $H_{*}\left(K_{0}\right)$ and an additive basis for this is $D=\left\{k_{\gamma}\right\}=\left\{x_{i}^{\epsilon_{i}} y_{j}^{\eta_{j}}\right\}$ where $\epsilon_{i}$ and $\eta_{j}$ are equal to 0 or 1 , so an additive basis for $H_{*}\left(G_{\lambda}\right)$ will contain all elements of this form. To prove the theorem in the case $i=0$ we need to show that the set $B^{\prime \prime}=\left\{s_{0}\left(c_{\alpha}\right) \cdot k_{\gamma}\right\}$ where $s_{0}\left(c_{\alpha}\right) \in B^{\prime}$ and $k_{\gamma} \in D$ is an additive basis of $H_{*}\left(G_{\lambda}\right)$. We start by proving that these elements generate additively $H_{*}\left(G_{\lambda}\right)$. Suppose we have an element $a \in H_{*}\left(G_{\lambda}\right)$. From Proposition 2.10 and Lemma 2.7 it is known that every element in $H_{*}\left(G_{\lambda}\right)$ is a sum of elements of the form (7). Therefore

$$
a=\sum_{\alpha} w_{J_{\alpha}} t^{\epsilon_{\alpha}} x_{i_{\alpha}}^{\epsilon_{i}} y_{j_{\alpha}}^{\eta_{j}}
$$

It is also known that $x_{i_{\alpha}}^{\epsilon_{i}} y_{j_{\alpha}}^{\eta_{j}}$ is in $D$ and if $w_{J_{\alpha}} t^{\epsilon_{\alpha}}$ is not in $B^{\prime}$ we can write it as sum of elements in $B^{\prime}$. Thus $a$ is a sum of elements in $B^{\prime \prime}$.

The next step is to show that the elements in $B^{\prime \prime}$ are linearly independent. We know that for a fixed degree $d$, the dimension of $H_{d}\left(G_{\lambda}\right)$ is given by

$$
\sum_{l=0}^{d} \operatorname{dim} H_{l}\left(U_{0}\right) \times \operatorname{dim} H_{d-l}\left(K_{0}\right),
$$

because of the vector space isomorphism (5). But this is precisely the number of elements in $B^{\prime \prime}$ of degree $d$. So they must be linearly independent otherwise their span would not be the space $H_{*}\left(G_{\lambda}\right)$. This means that the set $B^{\prime \prime}=$ $\left\{s_{0}\left(c_{\alpha}\right) \cdot k_{\gamma}\right\}$ defined above is an additive basis for $H_{*}\left(G_{\lambda}\right)$. Therefore $\varphi_{0}$ is an isomorphism.

In the case $i=1, \varphi_{1}$ maps $c \otimes k$ to $s_{1}(c) . k$ and this is not an element in the form (7). However we can prove an analogous result to Lemma 2.7 stating that any word in the $x_{i}, y_{j}, t$ is a sum of elements of the form $w_{I} x_{i}^{\epsilon_{i}} t y_{j}^{\eta_{j}}$. This is clear because $w_{I} t x_{i} y_{j}=w_{I} x_{i} t y_{j}+w_{I} w_{i} t y_{j}$ for all $I, i$ and $j$. Repeating the steps for the case $i=0$ and using isomorphism (6) instead of isomorphism (5) it follows easily that $\varphi_{1}$ is also an isomorphism. 
We are now in position to calculate the algebra structure on $H_{*}\left(G_{\lambda}\right)$.

Theorem 2.12 If $0<\lambda \leq 1$ then

$$
H_{*}\left(G_{\lambda} ; \mathbb{Z}_{2}\right)=\Lambda\left(y_{1}, y_{2}\right) \otimes \mathbb{Z}_{2}\left\langle t, x_{1}, x_{2}\right\rangle / R
$$

where $\operatorname{deg} y_{i}=\operatorname{deg} x_{i}=i, \operatorname{deg} t=1$ and $R$ is the set of relations $\left\{t^{2}=x_{i}^{2}=\right.$ $\left.0, x_{1} x_{2}=x_{2} x_{1}\right\}$.

Proof We already know from Proposition 2.10 that the generators of the Pontryagin ring are $t, x_{i}, y_{j}$. Therefore it is sufficient to prove that the only relations between them are the ones in $R$, the commutativity of $y_{i}$ with $x_{i}$ and $t$ plus the ones on $y_{i}$ coming from the definition of an exterior algebra $\Lambda\left(y_{1}, y_{2}\right)$. We also know from Lemma 2.7, assuming only these relations, that any word in these generators is a sum of elements of the form (7). Thus if we prove that this set of elements give an additive basis of $H_{*}\left(G_{\lambda}\right)$ we prove that there are no more relations between the generators $t, x_{i}, y_{i}$, because the existence of another relation would give rise to one between the elements of the form (7) and they would not be linearly independent. We will prove that by induction. The induction hypothesis is that up to dimension $d-1$ elements of form (7) are linearly independent, thus there are no relations between them up to dimension $d-1$. Suppose there was one of minimal degree $d$ in $H_{d}\left(G_{\lambda}\right)$. The first step is to show that it would be between the $w_{i}^{\prime} s$ only. Assume it was given by a finite sum of the type

$$
\sum_{k} w_{I_{k}} A_{k}=0
$$

where $w_{I_{k}}$ is a word on the $w_{i}^{\prime} s$ and $A_{k}=t^{\epsilon_{k}} b_{k}$ where $b_{k}$ is an element in $H_{*}\left(K_{0}\right)$ and $\epsilon_{k}$ equals 0 or 1 . Then from Proposition 2.11 with $i=0$ it follows that we must have

$$
\sum_{k} w_{I_{k}} t^{\epsilon_{k}} \otimes b_{k}=0
$$

We can group together the terms in which $b_{k}$ is the same, thus we can write the relation as

$$
\sum_{k}\left(\sum_{l_{k}} w_{l_{k}} t^{\epsilon_{l}}\right) \otimes b_{k}=0
$$

where now $b_{k}$ runs over a set of basis elements of $H_{*}\left(K_{0}\right)$. This implies that we have a relation of the type

$$
\sum_{l} w_{I_{l}} t^{\epsilon_{l}}=0
$$

Geometry 83 Topology, Volume 6 (2002) 
Using Proposition 2.11 with $i=1$ we show that it is between the $w_{i}^{\prime} s$, because

$$
\sum_{l} w_{I_{l}} \otimes t^{\epsilon_{l}}=\sum_{l^{\prime}} w_{I_{l^{\prime}}} \otimes t+\sum_{l^{\prime \prime}} w_{I_{l^{\prime \prime}}} \otimes 1=0
$$

implies

$$
\sum_{l^{\prime}} w_{I_{l^{\prime}}}=0 \text { and } \sum_{l^{\prime \prime}} w_{I_{l^{\prime \prime}}}=0
$$

A relation in the $w_{i}^{\prime} s$ projects, under the map $p_{0 *}$, to one on the $v_{i}{ }^{\prime} s$ in $H_{d}\left(U_{0}\right)$. Using isomorphism (1) this would give a relation in degree $d-1$ between the $u_{i}{ }^{\prime} s$ and $x_{i}^{\prime} s$ in $H_{d-1}\left(U_{1}\right)$. But this contradicts the induction hypothesis because such relation implies one in $H_{*}\left(G_{\lambda}\right)$ with $*$ at most equal to $d-1$.

The next corollary is an immediate consequence of the proof of the theorem.

Corollary 2.13 The Pontryagin ring $H_{*}\left(G_{\lambda}\right)$ contains a free noncommutative ring on 3 generators, namely $w_{1}, w_{2}, w_{3}$.

We proved also the following proposition:

Proposition 2.14 An additive basis for $H_{*}\left(G_{\lambda}\right)$ is given by

$$
w_{I} t^{\epsilon_{t}} x_{i}^{\epsilon_{i}} y_{j}^{\eta_{j}}
$$

where $\epsilon_{t}, \epsilon_{i}, \eta_{j}=0$ or $1, I=\left(i_{1}, \ldots, i_{n}\right)$ and $i, j=1,2$ or $3\left(x_{3}=x_{1} x_{2}, y_{3}=\right.$ $\left.y_{1} y_{2}\right)$.

\subsection{Relation between cohomology and homology}

Establishing the vector space isomorphisms (3) and (4) does not imply that we have algebra isomorphisms on cohomology. That is proved in the next lemma.

Lemma 2.15 The following isomorphisms hold as algebra isomorphisms:

$$
H^{*}\left(G_{\lambda}\right) \cong H^{*}\left(U_{i}\right) \otimes H^{*}\left(K_{i}\right) \text { with } i=0,1
$$

Proof The proof is based in the argument used by Abreu in [1] with some necessary changes. $H^{*}\left(G_{\lambda}\right)$ has subalgebras $p_{i}^{*}\left(H^{*}\left(U_{i}\right)\right) \cong H^{*}\left(U_{i}\right)$. From Theorem 2.3 we know that $\operatorname{Map}_{1}\left(S^{2}\right)$ is homotopy equivalent to $S O(3) \times \Omega$ where $\Omega$ denotes the universal covering space of $\operatorname{Map}_{1 *}\left(S^{2}\right)$. Therefore we have a map $\operatorname{Map}_{1}\left(S^{2}\right) \times \operatorname{Map}_{1}\left(S^{2}\right) \rightarrow S O(3) \times S O(3)$. The composite of $G_{\lambda} \rightarrow$ $\operatorname{Map}_{1}\left(S^{2}\right) \times \operatorname{Map}_{1}\left(S^{2}\right)$ with the previous map gives us a map $p: G_{*} \rightarrow K_{0}$. 
Thus $H^{*}\left(G_{\lambda}\right)$ has a subalgebra $p^{*}\left(H^{*}\left(K_{0}\right)\right) \cong H^{*}\left(K_{0}\right)$. Composing these inclusions of $H_{*}\left(U_{0}\right)$ and $H^{*}\left(K_{0}\right)$ as subalgebras of $H^{*}\left(G_{\lambda}\right)$ with cup product multiplication in $H^{*}\left(G_{\lambda}\right)$ we get a map

$$
\nu_{0}: H^{*}\left(U_{0}\right) \otimes H^{*}\left(K_{0}\right) \rightarrow H^{*}\left(G_{\lambda}\right) .
$$

$\nu_{0}$ is an algebra homomorphism because $H^{*}\left(G_{\lambda}\right)$ is commutative and it is compatible with filtrations (the obvious one on $H_{*}\left(U_{0}\right) \otimes H^{*}\left(K_{0}\right)$ and the filtration $F$ on $H^{*}\left(G_{\lambda}\right)$ coming from the fibration on the left in $\left.(2)\right)$. The degeneration of the spectral sequence at the $E_{2}$-term implies that $\nu_{0}$ is an algebra isomorphism. This proves isomorphism (11) in the case $i=0$. For the case $i=1$ note that the map $i_{1}^{*}: H^{*}\left(G_{\lambda}\right) \rightarrow H^{*}\left(K_{1}\right)$ is surjective, so there are $\hat{t}$ and $\hat{y}$ in $H^{*}\left(G_{\lambda}\right)$ such that $i_{1}^{*}(\hat{t})$ and $i_{1}^{*}(\hat{y})$ generate the ring $H^{*}\left(K_{1}\right)$, where $i_{1}^{*}(\hat{t})$ is the generator of the cohomology of $S^{1}$ and $\hat{t}$ is such that $\hat{t}\left(x_{1}\right)=0 . i_{1}^{*}(\hat{y})$ is the generator of the cohomology of the $S O(3)$ factor. Now we need to prove that $\hat{t}^{2}=0$ in $H^{*}\left(G_{\lambda}\right)$ in order to claim that the subalgebra of $H^{*}\left(G_{\lambda}\right)$ generated by $\hat{t}$ and $\hat{y}$ is isomorphic to $H^{*}\left(K_{1}\right)$.

Lemma $2.16 \quad \hat{t}^{2}=0$ in $H^{*}\left(G_{\lambda}\right)$

Proof Using isomorphisms (1), (5) and (6) we can show that the rank of $H_{2}\left(G_{\lambda}\right)$ is 6 . But in $H_{2}\left(G_{\lambda}\right)$ the cycles $x_{2}, y_{2}, t x_{1}, t y_{1}, x_{1} y_{1}, w_{1}$ are linearly independent. We will show that $\hat{t}^{2}$ evaluated on all these classes is 0 . The only one at which is not obviously 0 is $w_{1}$. Let the map $\alpha: S^{2}=S^{1} \times S^{1} / S^{1} \vee S^{1} \rightarrow$ $G_{\lambda}$ represent the $2-$ cycle $w_{1}$. Then $\hat{t}^{2}\left(w_{1}\right)=\alpha^{*}\left(\hat{t}^{2}\right)\left[S^{2}\right]=\left(\alpha^{*}(\hat{t})\left[S^{2}\right]\right)^{2}$ and this vanishes because $w_{1}$ is a spherical class, ie, $\alpha^{*}(\hat{t}) \in H^{1}\left(S^{2}\right)=0$.

Again composing these inclusions of $H^{*}\left(K_{1}\right)$ and $H_{*}\left(U_{1}\right)$ as subalgebras of $H^{*}\left(G_{\lambda}\right)$ with cup product multiplication we get a map

$$
\nu_{1}: H^{*}\left(U_{1}\right) \otimes H^{*}\left(K_{1}\right) \rightarrow H^{*}\left(G_{\lambda}\right)
$$

which is an algebra isomorphism.

Remark 2.17 From the isomorphisms in the previous Lemma and in Proposition 2.11 we might be tempted to think that the diagram

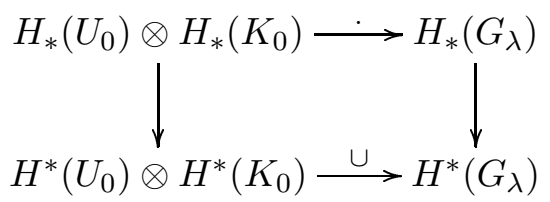


commutes, where the vertical arrows are given by taking a basis $b_{i}$ of $H_{*}\left(U_{0}\right)$ or $H_{*}\left(G_{\lambda}\right)$ to its dual basis. Actually this diagram does not commute. To see that we can consider the following example.

We have

$$
\left\langle\hat{w}_{1} \cup \widehat{x_{1} y_{2}}, w_{1} x_{1} y_{2}\right\rangle=1
$$

and if diagram (12) was commutative then the cup product $\hat{w}_{1} \cup \widehat{x_{1} y_{2}}$ evaluated at all other elements would be 0 . But it is not difficult to verify that we have

$$
\left\langle\hat{w}_{1} \cup \widehat{x_{1} y_{2}}, w_{2} y_{2}\right\rangle=1 \text {. }
$$

\subsubsection{The cohomology ring with $\mathbb{Z}_{2}$ coefficients}

Since $H^{*}\left(G_{\lambda} ; \mathbb{Z}_{2}\right)$ is a Hopf algebra we can use the classification theorem of commutative Hopf algebras over a field of characteristic 2 . It says that $H^{*}\left(G_{\lambda} ; \mathbb{Z}_{2}\right)$ is a tensor product of the type

$$
\left.\left(\underset{\alpha}{\otimes} \mathbb{Z}_{2}\left[x_{\alpha}\right] / x_{\alpha}^{h\left(x_{\alpha}\right)}\right) \otimes \underset{\beta}{\otimes} \mathbb{Z}_{2}\left[x_{\beta}\right]\right)
$$

where $h\left(x_{\alpha}\right)$ is a power of 2 (see [13] for a proof of this structure theorem).

In fact we can prove the following proposition.

Proposition 2.18 If $0<\lambda \leq 1, H^{*}\left(G_{\lambda} ; \mathbb{Z}_{2}\right)$ is isomorphic, as an algebra, to a tensor product of an exterior algebra over $\mathbb{Z}_{2}$ with an infinite number of generators and a truncated polynomial algebra with two generators of multiplicative order 4 .

Proof Note that Lemma 2.15 shows that $H^{*}\left(G_{\lambda} ; \mathbb{Z}_{2}\right)$ is the tensor product of the algebras $H^{*}\left(U_{0}\right)$ and $H^{*}\left(K_{0}\right)$. Since $H^{*}(S O(3))=\mathbb{Z}_{2}\left[\hat{x_{1}}\right] /\left\{{\hat{x_{1}}}^{4}=0\right\}$ we can conclude that $H^{*}\left(K_{0}\right)$ is a commutative algebra with two generators of multiplicative order 4. Next we show that $H^{*}\left(U_{0}\right)$ has an infinite number of generators and all have order 2 . This proves the proposition. As we stated in Remark 2.9, the isomorphism $\phi: H^{p+1}\left(U_{0}\right) \rightarrow H^{p}\left(U_{1}\right)$ is the composite of the restriction

$$
i^{*}: H^{*}\left(U_{0}\right) \longrightarrow H^{*}\left(\partial \mathcal{N}_{U_{1}}\right)
$$

with integration over the fiber of the projection $\pi: \partial \mathcal{N}_{U_{1}} \rightarrow U_{1}$ of the fibration (8). Since integration over the fiber kills the elements of $\pi^{*}\left(H^{*}\left(U_{1}\right)\right)$, for each $0 \neq v \in H^{p}\left(U_{0}\right)$ there is $k \in \mathbb{Z}_{2}$ such that

$$
i^{*}(v)=\hat{t} \cup \pi^{*}(u)+k \pi^{*}\left(u^{\prime}\right)
$$


where $u \in H^{p-1}\left(U_{1}\right), u^{\prime} \in H^{p}\left(U_{1}\right)$ and $\phi(v)=u$. Therefore we have $i^{*}\left(v^{2}\right)=$ $2 \hat{t} \cup \pi^{*}\left(u u^{\prime}\right)+k^{2} \pi^{*}\left(u^{\prime 2}\right)=k^{2} \pi^{*}\left(u^{\prime 2}\right)$, because $\hat{t}^{2}=0$ (proved in Lemma 2.16). Thus $\phi\left(v^{2}\right)=0$. Since $\phi$ is an isomorphism we get $v^{2}=0$. From knowing that all generators in $H^{*}\left(U_{0}\right)$ have multiplicative order 2 it follows that $H^{*}\left(U_{0}\right)$ must have an infinite number of generators, just by comparing the dimensions $\operatorname{dim} H^{p}\left(U_{0}\right)=\operatorname{dim} H_{p}\left(U_{0}\right)$ for each $p$. Recall that Theorem 2.12 implies that $H_{*}\left(G_{\lambda} ; \mathbb{Z}_{2}\right)$ contains a free noncommutative ring on 3 generators that projects to non-zero elements in $H_{*}\left(U_{0}, \mathbb{Z}_{2}\right)$ and the dimensions $\operatorname{dim} H_{p}\left(U_{0}\right)$ increase as $p$ increases.

Remark 2.19 In the rational case Abreu proved in [1] that the cohomology ring $H^{*}\left(U_{0}, \mathbb{Q}\right)$ contains a generator in dimension 4 of infinite order. The previous result does not contradict this fact, but shows that $H_{*}\left(G_{\lambda} ; \mathbb{Z}\right)$ contains a divided polynomial algebra. We will confirm this fact in Section 3 when we compute the homotopy type of $G_{\lambda}$.

\section{Homotopy type of $G_{\lambda}$}

In this section we will show that $G_{\lambda}$ is homotopy equivalent to the product $X=L \times S^{1} \times S O(3) \times S O(3)$ where $L=\Omega \Sigma\left(S^{1} \times S O(3)\right)$. We start by giving some considerations about torsion in $H_{*}\left(G_{\lambda} ; \mathbb{Z}\right)$. In the second subsection we explain why we consider the loop space $L$ and the last subsection is devoted to the construction of the homotopy equivalence between $G_{\lambda}$ and $X$.

\subsection{Torsion in $H_{*}\left(G_{\lambda} ; \mathbb{Z}\right)$}

We can repeat the argument used in Section 2 to compute the Pontryagin rings $H_{*}\left(G_{\lambda} ; \mathbb{Q}\right)$ and $H_{*}\left(G_{\lambda} ; \mathbb{Z}_{p}\right)$, with $\mathbb{Q}$ and $\mathbb{Z}_{p}$ coefficients, with $p$ prime and $\neq 2$. In this case the homology of $S O(3)$ is given by a single generator in dimension 3. Therefore it is easy to see that the generators of the homology ring of $G_{\lambda}$, in this case, are simply $t, x_{3}$ and $y_{3}$, where $t^{2}=x_{3}^{2}=y_{3}^{2}=0, y_{3}$ commutes with $x_{3}$ and $t$, but $t$ does not commute with $x_{3}$. Thus we obtain a theorem analogous to Theorem 2.12:

Theorem 3.1 If $0<\lambda \leq 1$ then

$$
H_{*}\left(G_{\lambda} ; F\right) \cong \Lambda\left(y_{3}\right) \otimes F\left\langle t, x_{3}\right\rangle / R
$$

where $\operatorname{deg} y_{3}=3, R$ is the set of relations $\left\{t^{2}=x_{3}^{2}=0\right\}$ and $F$ is the field $\mathbb{Q}$ or $\mathbb{Z}_{p}$ with $p \neq 2$. 
Moreover an additive basis for the homology is given by elements of the form

$$
w_{3}^{k} t^{\epsilon t} x_{3}^{\epsilon} y_{3}^{\eta},
$$

where $\epsilon_{t}, \epsilon, \eta=0$ or $1, k \in \mathbb{N}$ and $w_{3}$ is obtained as the commutator of $x_{3}$ and $t$. Since the results are the same if we consider $\mathbb{Q}$ coefficients or $Z_{p}$ coefficients with $p \neq 2$ we can conclude that $H_{*}\left(G_{\lambda} ; \mathbb{Z}\right)$ has no $p$-torsion if $p \neq 2$.

\subsection{The James construction}

We proved in Section 2 that $H_{*}\left(G_{\lambda} ; \mathbb{Z}_{2}\right)$ contains a free non-commutative algebra on 3 generators in dimensions 2, 3 and 4 . Now the aim is to find an $H$-space $L$ such that the homology ring $H_{*}\left(L ; \mathbb{Z}_{2}\right)$ is isomorphic to this algebra $\mathbb{Z}_{2}\left\langle w_{1}, w_{2}, w_{3}\right\rangle$ where $w_{i}$ is in dimension $i+1$. In order to find such space we will use the James construction that we describe next.

For a pointed topological space $(X, *)$, let $J_{k}(X)=X^{k} / \sim$ where

$$
\left(x_{1}, \ldots, x_{j-1}, *, x_{j+1}, \ldots, x_{k}\right) \sim\left(x_{1}, \ldots, x_{j-1}, x_{j+1}, *, \ldots, x_{k}\right) .
$$

The James construction on $X$, denoted $J(X)$ is defined by

$$
J(X)=\lim _{\vec{k}} J_{k}(X),
$$

where $J_{k}(X) \subset J_{k+1}(X)$ by adding $*$ in the last component. There is a canonical inclusion $X=J_{1}(X) \hookrightarrow J(X) . J(X)$ is a topological monoid and any map from $X$ to a topological monoid $M$ extends uniquely to a morphism $J(X) \rightarrow M$ of topological monoids. That is, $X \hookrightarrow J(X)$ is universal with respect to maps from $X$ to topological monoids, ie, if $f: X \rightarrow M$ is given there is a unique $\tilde{f}$ such that the following diagram commutes:

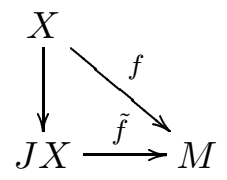

$\tilde{f}$ is defined by $\tilde{f}\left(x_{1}, \ldots, x_{k}\right)=f\left(x_{1}\right) \ldots f\left(x_{k}\right)$. From the definition, $J^{k} X / J^{k-1} X=X \wedge X \wedge \ldots \wedge X$ and since we have a filtration

$$
J X \supset \ldots \supset J^{k} X \supset J^{k-1} X \supset \ldots
$$

applying the Künneth Theorem we conclude the following (see [16]): 


\section{Theorem 3.2}

$$
H_{*}\left(J^{k} X ; \mathbb{Z}_{2}\right)=H_{*}\left(J^{k-1} X ; \mathbb{Z}_{2}\right) \oplus H_{*}\left(X \wedge X \wedge \ldots \wedge X ; \mathbb{Z}_{2}\right)
$$

and

$$
\tilde{H}_{*}\left(J X ; \mathbb{Z}_{2}\right)=\oplus_{k}\left(\tilde{H}_{*}\left(X ; \mathbb{Z}_{2}\right)\right)^{\otimes_{k}}=T\left(\tilde{H}_{*}\left(X ; \mathbb{Z}_{2}\right)\right)
$$

where given a vector space $H, T(H)$ is the tensor algebra on $H$ and the last isomorphism is an isomorphism of Pontryagin rings.

Now note the following theorem (see proof in [14]).

Theorem 3.3 (James) If $X$ has the homotopy type of a connected $C W$ complex then $J X$ and $\Omega \Sigma X$ are homotopy equivalent.

The Theorems 3.2 and 3.3 imply that

$$
\tilde{H}_{*}\left(\Omega \Sigma X ; \mathbb{Z}_{2}\right) \cong T\left(\tilde{H}_{*}\left(X ; \mathbb{Z}_{2}\right)\right)
$$

so, in particular, if $X=S^{1} \wedge S O(3)$ we get

$$
\tilde{H}_{*}\left(\Omega \Sigma\left(S^{1} \wedge S O(3)\right) ; \mathbb{Z}_{2}\right) \cong T\left(\tilde{H}_{*}\left(S^{1} \wedge S O(3) ; \mathbb{Z}_{2}\right)\right) \cong \mathbb{Z}_{2}\left\langle w_{1}, w_{2}, w_{3}\right\rangle,
$$

where $w_{1}, w_{2}, w_{3}$ are generators in dimension $2,3,4$ respectively. So we see that the homology with $\mathbb{Z}_{2}$ coefficients of this space is isomorphic to a subalgebra of $H_{*}\left(G_{\lambda} ; \mathbb{Z}_{2}\right)$.

\subsection{The homotopy equivalence}

The following result is well known: see [6], Corollary 3.37.

Proposition 3.4 A map $f: X \rightarrow Y$ induces isomorphisms on homology with $\mathbb{Z}$ coefficients iff it induces isomorphisms on homology with $\mathbb{Q}$ and $\mathbb{Z}_{p}$ coefficients for all primes $p$.

We now define the map $f$ from $X=\Omega \Sigma\left(S^{1} \times S O(3)\right) \times S^{1} \times S O(3) \times S O(3)$ to $G_{\lambda}$ that induces isomorphisms on homology with $\mathbb{Q}$ and $\mathbb{Z}_{p}$ coefficients, for all primes $p$. We have an inclusion map

$$
i: S^{1} \times S O(3) \rightarrow G_{\lambda}
$$

given by

$$
(x, y) \mapsto i_{1}(x) i_{0}(y) i_{1}(x)^{-1} i_{0}(y)^{-1}
$$


where $i_{0}$ and $i_{1}$ are the inclusions defined in Section 2. More precisely, in this formula $i_{1}$ is the restriction of the inclusion $K_{1} \hookrightarrow G_{\lambda}$ to the $S^{1}$ factor and $i_{0}$ is the restriction of the inclusion $K_{0} \hookrightarrow G_{\lambda}$ to the first $S O(3)$ factor. The restriction to $S^{1} \vee S O(3)$ of $i$ is the identity so there is an induced map

$$
h: S^{1} \wedge S O(3) \rightarrow G_{\lambda} .
$$

This map induces the right correspondence between generators in homology

$$
h_{*}: H_{*}\left(S^{1} \wedge S O(3) ; \mathbb{Z}_{2}\right) \rightarrow H_{*}\left(G_{\lambda} ; \mathbb{Z}_{2}\right),
$$

this meaning that the three generators of $H_{*}\left(S^{1} \wedge S O(3) ; \mathbb{Z}_{2}\right)$ are mapped to $w_{1}, w_{2}, w_{3} \in H_{*}\left(G_{\lambda} ; \mathbb{Z}_{2}\right)$, because as we saw before these generators in $H_{*}\left(G_{\lambda} ; \mathbb{Z}_{2}\right)$ are obtained as commutators of the form (13). Moreover there is a unique map $\tilde{h}$ that extends $h$ to $\Omega \Sigma\left(S^{1} \wedge S O(3)\right)$ as we explained in Section 3.2. Therefore the map

$$
\tilde{h}_{*}: H_{*}\left(\Omega \Sigma\left(S^{1} \wedge S O(3)\right) ; \mathbb{Z}_{2}\right) \rightarrow H_{*}\left(G_{\lambda} ; \mathbb{Z}_{2}\right)
$$

takes the generators of $H_{*}\left(\Omega \Sigma\left(S^{1} \wedge S O(3)\right) ; \mathbb{Z}_{2}\right)$ to the elements $w_{1}, w_{2}, w_{3}$ in $H_{*}\left(G_{\lambda} ; \mathbb{Z}_{2}\right)$. Now consider the map $f: L \times S^{1} \times S O(3) \times S O(3) \rightarrow G_{\lambda}$ given by

$$
(w, t, y, x) \mapsto \tilde{h}(w) i_{1}(t, y) i_{0}(x),
$$

where $w \in L=\Omega \Sigma\left(S^{1} \wedge S O(3)\right)$.

Lemma 3.5 The map $f$ defined above induces isomorphisms on homology with $\mathbb{Q}$ and $\mathbb{Z}_{p}$ coefficients for all primes $p$.

Proof The map $f$ restricted to $S^{1} \times S O(3)$ or the second $S O(3)$ factor is just the inclusion in $G_{\lambda}$. Moreover, using the Künneth formula for homology with coefficients in a field $\mathrm{F}$, we get

$$
H_{n}(X ; F) \cong \underset{p+q+l=n}{\oplus} H_{p}(L ; F) \otimes H_{q}\left(S^{1} \times S O(3) ; F\right) \otimes H_{l}(S O(3) ; F) .
$$

Let $F$ be $\mathbb{Q}$ or $\mathbb{Z}_{p}$ with $p \neq 2$. Note that in this case $H_{*}(S O(3) ; F)$ has only a generator in dimension 3 . Therefore an additive basis for $H_{*}\left(G_{\lambda} ; F\right)$ is given by

$$
w_{3}^{k} t^{\epsilon} x_{3}^{\epsilon} y_{3}^{\eta}
$$

where $\epsilon_{t}, \epsilon, \eta=0$ or 1 . Thus comparing equation (16) and an additive basis for $H_{*}\left(G_{\lambda} ; F\right)$ we conclude that the homology groups of $X$ and $G_{\lambda}$ are the same. We just need to show that $f$ induces those isomorphisms. The elements $t, x_{3}$ 
and $y_{3}$ are the images of the generators of $H_{*}\left(S^{1} \times S O(3) ; F\right)$ and $H_{*}(S O(3) ; F)$ under the injective maps

$$
i_{1 *}: H_{*}\left(S^{1} \times S O(3) ; F\right) \rightarrow H_{*}\left(G_{\lambda} ; F\right)
$$

and

$$
i_{0 *}: H_{*}(S O(3) ; F) \rightarrow H_{*}\left(G_{\lambda} ; F\right)
$$

induced by inclusions $i_{0}$ and $i_{1}$. On the other hand the restriction of $f$ to $L$ is given by the map $\tilde{h}$ and we know that $\tilde{h}_{*}$ maps the 4-dimensional generator of

$$
H_{*}\left(\Omega \Sigma\left(S^{1} \wedge S O(3)\right) ; F\right) \cong T\left(\tilde{H}_{*}\left(S^{1} \wedge S O(3) ; F\right)\right) \cong F\left[w_{3}\right]
$$

to the element in $H_{4}\left(G_{\lambda} ; F\right)$ obtained as the Samelson product of $t$ and $x_{3}$. This proves that $f$ induces an isomorphism in homology with $\mathbb{Q}$ and $\mathbb{Z}_{p}$ coefficients for all primes $p$ with $p \neq 2$.

If $F=\mathbb{Z}_{2}$ then an additive basis for $H_{*}\left(G_{\lambda} ; \mathbb{Z}_{2}\right)$ is given by the set of elements of the form (7). It follows from equation (16) that the homology groups $H_{*}\left(G_{\lambda} ; \mathbb{Z}_{2}\right)$ and $H_{*}\left(X ; \mathbb{Z}_{2}\right)$ are isomorphic. The elements $t, y_{1}, y_{2}$ are the images of the generators of $H_{*}\left(S^{1} \times S O(3) ; \mathbb{Z}_{2}\right)$ and $x_{1}, x_{2}$ are the images of the generators of $H_{*}\left(S O(3) ; \mathbb{Z}_{2}\right)$. In this case the map $\tilde{h}_{*}$ stated in (14) takes the generators of $H_{*}\left(\Omega \Sigma\left(S^{1} \wedge S O(3)\right) ; \mathbb{Z}_{2}\right)$ to the elements $w_{1}, w_{2}, w_{3}$ which are the three generators of the free noncommutative subalgebra of $H_{*}\left(G_{\lambda} ; \mathbb{Z}_{2}\right)$. Therefore we get another isomorphism in homology.

Since the conditions of Proposition 3.4 are satisfied we can conclude that the map $f$ defined in (15) induces isomorphisms on homology with $\mathbb{Z}$ coefficients. Using the fact the $G_{\lambda}$ and $X$ are both $H$-spaces it follows that $\pi_{1}$ acts trivially on all $\pi_{n}{ }^{\prime} s$ in each one of the spaces (see [16], pp 119). This allow us to apply the following corollary of Whitehead's theorem ([6] proposition 4.48):

Corollary 3.6 If $X$ and $Y$ are abelian $C W$-complexes (i. e. $\pi_{1}$ acts trivially on all $\pi_{n}{ }^{\prime} s$ ) then a map $f: X \rightarrow Y$ that induces isomorphisms in homology is a homotopy equivalence.

Therefore we have proved our main theorem:

Theorem 3.7 If $0<\lambda \leq 1, G_{\lambda}$ is homotopy equivalent to the product $\Omega \Sigma\left(S^{1} \wedge S O(3)\right) \times S^{1} \times S O(3) \times S O(3)$. 
Remark 3.8 Although the spaces $G_{\lambda}$ and $\Omega \Sigma\left(S^{1} \wedge S O(3)\right) \times S^{1} \times S O(3) \times$ $S O(3)$ are homotopy equivalent the above homotopy equivalence is not an $H-$ map, because it does not preserve the product structure. This can be seen by comparing the Pontryagin products on integral homology. It would be an interesting question to find an easily understandable $H$-space with a Pontryagin ring isomorphic to the one of $G_{\lambda}$.

\section{References}

[1] M Abreu, Topology of symplectomorphism groups of $S^{2} \times S^{2}$, Invent. Math. 131 (1998) 1-23

[2] M Abreu, D McDuff, Topology of symplectomorphism groups of rational ruled surfaces, J. Amer.Math. Soc. 13 (2000) 971-1009

[3] W Browder, Torsion in H-spaces, Annals of Math. 74 (1961) 24-51

[4] M Gromov, Pseudo holomorphic curves in symplectic manifolds, Invent. Math. 82 (1985) 307-347

[5] V Hansen, The space of self maps on the 2-sphere. In Groups of selfequivalences and related topics (Montreal,PQ,1988), Lecture Notes in Math. 1425, Springer (1990) 40-47

[6] A Hatcher, Algebraic Topology, Cambridge University Press (2001)

[7] K Kodaira, Complex Manifolds and Deformation of Complex Structures, Springer-Verlag (1986)

[8] J McCleary, User's Guide to Spectral Sequences, Mathematics Lecture Series 12 , Publish or Perish (1985)

[9] D McDuff, Examples of symplectic structures, Invent. Math. 89 (1987) 13-36

[10] D McDuff, Almost complex structures on $S^{2} \times S^{2}$, Duke math. Journal 101 (2000) $135-177$

[11] D McDuff, D A Salamon, J-holomorphic curves and quantum cohomology, University Lectures Series 6, American Mathematical Society (1994)

[12] D McDuff, D A Salamon, Introduction to Symplectic Topology, 2nd edition, Oxford University Press (1998)

[13] M Mimura, H Toda, Topology of Lie Groups, I and II, Translations of Mathematical Monographs 91, American Mathematical Society (1991)

[14] P Selick, Introduction to Homotopy Theory, Fields Institute Monographs 9, American Mathematical Society (1991)

[15] E Spanier, Algebraic Topology, McGraw-Hill (1996)

[16] G W Whitehead, Elements of Homotopy Theory, Springer-Verlag (1978) 